\title{
Construction and Validation of a Risk Prediction Model for Acute Kidney Injury in Patients Suffering from Septic Shock
}

\author{
Suru Yue, ${ }^{1,2}$ Shasha Li, ${ }^{1}$ Xueying Huang, ${ }^{1}$ Jie Liu, ${ }^{1}$ Xuefei Hou, ${ }^{1,2}$ Yufeng Wang, ${ }^{1,2}$ \\ and Jiayuan $\mathrm{Wu}\left(\mathbb{C}^{1,2}\right.$ \\ ${ }^{1}$ Clinical Research Service Center, The Affiliated Hospital of Guangdong Medical University, Zhanjiang, \\ 524001 Guangdong Province, China \\ ${ }^{2}$ Collaborative Innovation Engineering Technology Research Center of Clinical Medical Big Data Cloud Service in Medical \\ Consortium of West Guangdong Province, The Affiliated Hospital of Guangdong Medical University, Zhanjiang, \\ 524001 Guangdong Province, China
}

Correspondence should be addressed to Jiayuan Wu; wujiay@gdmu.edu.cn

Received 14 September 2021; Accepted 22 December 2021; Published 6 January 2022

Academic Editor: Yuzhen Xu

Copyright (c) 2022 Suru Yue et al. This is an open access article distributed under the Creative Commons Attribution License, which permits unrestricted use, distribution, and reproduction in any medium, provided the original work is properly cited.

Background. Acute kidney injury (AKI) is an important complication in critically ill patients, especially in sepsis and septic shock patients. Early prediction of AKI in septic shock can provide clinicians with sufficient information for timely intervention so that improve the patients' survival rate and quality of life. The aim of this study was to establish a nomogram that predicts the risk of AKI in patients with septic shock in the intensive care unit (ICU). Methods. The data were collected from the Medical Information Mart for Intensive Care III (MIMIC-III) database between 2001 and 2012. The primary outcome was AKI in the $48 \mathrm{~h}$ following ICU admission. Univariate and multivariate logistic regression analyses were used to screen the independent risk factors of AKI. The performance of the nomogram was evaluated according to the calibration curve, receiver operating characteristic (ROC) curve, decision curve analysis, and clinical impact curve. Results. A total of 2415 patients with septic shock were included in this study. In the training and validation cohort, 1091 (64.48\%) of 1690 patients and 475 (65.52\%) of 725 patients developed AKI, respectively. The predictive factors for nomogram construction were gender, ethnicity, congestive heart failure, diabetes, obesity, Simplified Acute Physiology Score II (SAPS II), angiotensin-converting enzyme inhibitors (ACEI) or angiotensin receptor blockers (ARBs), bilirubin, creatinine, blood urea nitrogen (BUN), and mechanical ventilation. The model had a good discrimination with the area under the ROC curve of 0.756 and 0.760 in the training and validation cohorts, respectively. The calibration curve for probability of AKI in septic shock showed optimal agreement between prediction by nomogram and actual observation. Decision curve and clinical impact curve analysis indicated that the nomogram conferred high clinical net benefit. Conclusion. The proposed nomogram can quickly and effectively predict the risk of AKI at an early stage in patients with septic shock in ICU, which can provide information for timely and efficient intervention in patients with septic shock in the ICU setting.

\section{Introduction}

Septic shock is a life-threatening severe disease caused by circulatory and cellular metabolic abnormalities; it affects $10 \%-$ $30 \%$ of patients in the intensive care unit (ICU) $[1,2]$. The mortality rate of septic shock is estimated to be $45 \%-63 \%$ [3]. Septic shock is the most common cause of acute kidney injury (AKI) in critically ill patients. The decreased renal blood flow, secondary tubular epithelial cell death, or acute tubular necrosis is the core mechanisms underlying AKI in septic shock patients $[4,5]$. The prevalence of AKI in patients suffering from septic shock was up to $60.47 \%$, and the mortality rate is as high as $62.1 \%[5,6]$. In addition, the development of AKI during septic shock increases the patients' mortality and prolongs the hospital stay $[7,8]$. However, AKI can be prevented in the early stage of septic shock because of the compensatory 


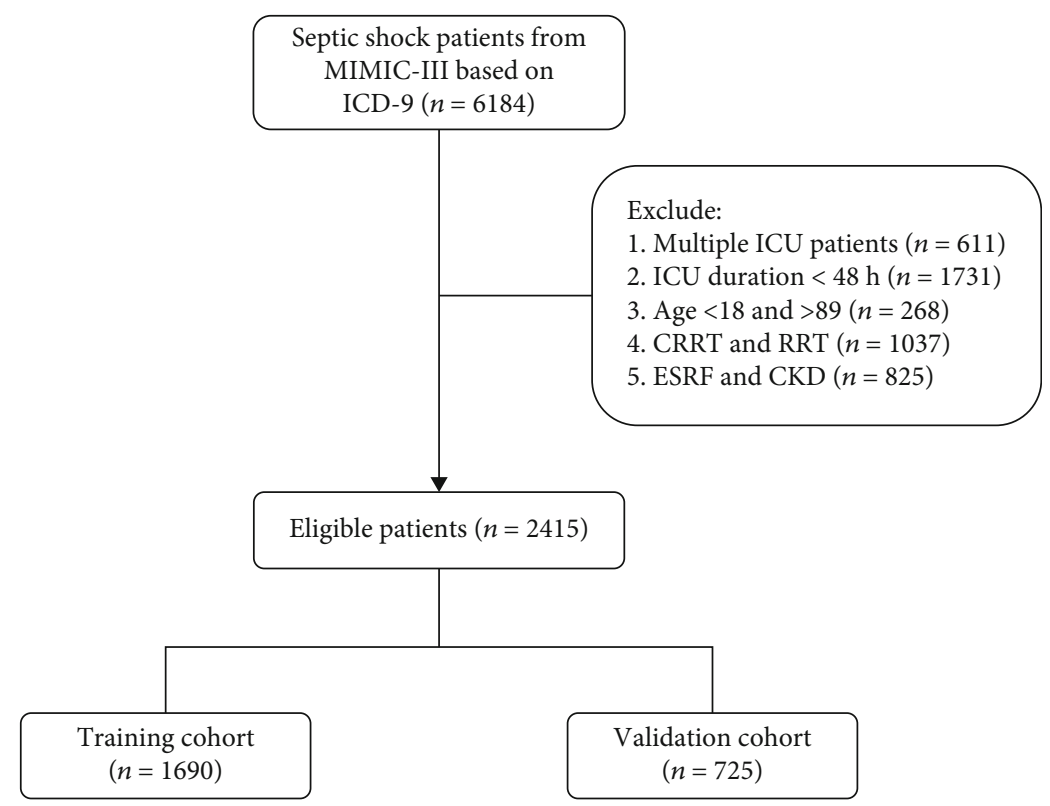

FIGURE 1: The flowchart of patient selection. MIMIC-III: Medical Information Mort for Intensive Care III; ICU: intensive care unit; CRRT: continuous renal replacement therapy.

and reverse recovery function of the kidneys [9]. Thus, a simple and convenient method can be used to quickly assess the risk of AKI in septic shock.

For the past decades, many researchers have focused on the risk factors of AKI in patients suffering from septic shock. Among them, blood or serum biomarkers, such as the delta neutrophil index (DNI), proenkephalin (PENK), urinary interleukin-18, urinary KIM-1, and neutrophil gelatinaseassociated lipocalin (NGAL), have attracted attention [10-14]. However, these indicators are difficult to apply to clinical practice due to the high cost and technical requirement. Moreover, several risk factors of AKI development in critically ill patients, including older age, obesity, mechanical ventilation, low white blood cell (WBC), and platelet counts, have been explored [15]. However, these indices are not stable when they solely acted as a single index due to the effects of confounding factors. Thus, it is more appropriated to incorporate them into a comprehensive model. A nomogram is a userfriendly tool with graphical representation that can be used to calculate the probability of a specific event for each individual. Compared with single indexes, nomograms can more accurately estimate the risk of AKI for individual patients by incorporating multiple risk factors. Recently, several nomograms have been developed for the prediction of AKI in many diseases. Deng et al. incorporated the information of 2917 patients with sepsis, including blood urea nitrogen (BUN), infusion volume, serum lactate, weight, serum chloride, body temperature, and age, to formulate a nomogram to predict AKI during the first $24 \mathrm{~h}$ of ICU stay, and the model showed excellent performance with a $C$ index of 0.80 [16]. A nomogram using the routine information in ICU was wellcalibrated and clinically useful for the prediction of AKI in patients with diabetic ketoacidosis [17]. However, nomograms for predicting AKI in patients suffering from septic shock are yet to be reported. As the high mortality rate of AKI in septic shock is partly due to a delay in diagnosis, identifying the septic shock patients at high risk of AKI can help clinicians take timely and effective intervention measures, reduce the mortality, and improve the quality of life. Therefore, this study is aimed at developing and validating a nomogram for predicting AKI in patients suffering from septic shock in the ICU.

\section{Methods}

2.1. Source of Data. The data of this study was extracted from the Medical Information Mort for Intensive Care III (MIMIC-III) database [18]. MIMIC-III is a large, free accessible intensive care database that contains the detailed information of more than 40,000 patients admitted to the critical care units in the Beth Israel Deaconess Medical Center (BIDMC) from 2001 to 2012, including demographic characteristics, monitoring vital signs, laboratory and microbiological examination, imaging examination, observation and recording of intake and output, drug treatment, length of stay, survival data, and discharge or death records. To apply for access to the database, we passed the protection of human research participant examination and obtained the certificate (No. 9983480). Structured Query Language (SQL) was used to extract all patients' information from the MIMIC-III database.

2.2. Participants. Inclusive criteria were as follows: (1) first admission to ICU and (2) diagnosis of septic shock upon admission. Exclusion criteria were as follows: (1) the length of stay in ICU was less than $48 \mathrm{~h},(2) \leq 18$ and $\geq 89$ years old, (3) underwent renal replacement therapy or continuous renal replacement therapy, (4) died within $48 \mathrm{~h}$ after ICU admission, and (4) diagnosed with end-stage renal disease 
TABLE 1: Baseline characteristics of the patients with septic shock.

\begin{tabular}{|c|c|c|c|c|}
\hline Characteristics & Total $(\mathrm{n}=2415)$ & Train cohort $(\mathrm{n}=1690)$ & Validation cohort $(\mathrm{n}=725)$ & $P$ value \\
\hline \multicolumn{5}{|l|}{ Demographic variables } \\
\hline Age (years) & $65(55-76)$ & $65(55-75)$ & $65(55-77)$ & 0.120 \\
\hline Gender, $n(\%)$ & & & & 0.850 \\
\hline Male & $1263(52.30)$ & $886(52.43)$ & $377(52.00)$ & \\
\hline Female & $1152(47.70)$ & $804(47.57)$ & $348(48.00)$ & \\
\hline Ethnicity, $n(\%)$ & & & & 0.550 \\
\hline Caucasian & $1796(74.37)$ & $1251(74.02)$ & $545(75.17)$ & \\
\hline Non-Caucasian & $619(25.63)$ & $439(25.98)$ & $180(24.83)$ & \\
\hline Obesity, $n(\%)$ & & & & 0.020 \\
\hline No & $2276(94.24)$ & $1580(93.49)$ & $696(96.00)$ & \\
\hline Yes & $619(5.76)$ & $110(6.51)$ & $29(4.00)$ & \\
\hline \multicolumn{5}{|l|}{ Comorbidities } \\
\hline Congestive heart failure, $n(\%)$ & & & & 0.350 \\
\hline No & $1556(64.43)$ & $1099(65.03)$ & $457(63.03)$ & \\
\hline Yes & $859(35.57)$ & $591(34.97)$ & $268(36.97)$ & \\
\hline Hypertension, $n(\%)$ & & & & 0.590 \\
\hline No & $1379(57.10)$ & $971(57.46)$ & $408(56.28)$ & \\
\hline Yes & $1036(42.90)$ & $719(42.54)$ & $317(43.72)$ & \\
\hline Diabetes, $n(\%)$ & & & & 0.660 \\
\hline No & $1677(69.44)$ & $1169(69.17)$ & $508(70.07)$ & \\
\hline Yes & $738(30.56)$ & $521(30.83)$ & $217(29.93)$ & \\
\hline \multicolumn{5}{|l|}{ Medications } \\
\hline Aminoglycoside, $n(\%)$ & & & & 0.750 \\
\hline No & $2266(93.83)$ & $1584(93.73)$ & $682(94.07)$ & \\
\hline Yes & $149(6.17)$ & $106(6.27)$ & $43(5.93)$ & \\
\hline Glycopeptide antibiotics, $n(\%)$ & & & & 0.230 \\
\hline No & $690(28.57)$ & $495(29.29)$ & $195(26.90)$ & \\
\hline Yes & $1725(71.43)$ & $1195(70.71)$ & $530(73.10)$ & \\
\hline NSAIDs, $n(\%)$ & & & & 0.950 \\
\hline No & $714(29.57)$ & $499(29.53)$ & $215(29.66)$ & \\
\hline Yes & $1701(70.43)$ & $1191(70.47)$ & $510(70.34)$ & \\
\hline Stain, $n(\%)$ & & & & 0.940 \\
\hline No & $1963(81.28)$ & $1373(81.24)$ & $590(81.38)$ & \\
\hline Yes & $452(18.72)$ & $317(18.76)$ & $135(18.62)$ & \\
\hline ACEI/ARBs, $n(\%)$ & & & & 0.820 \\
\hline No & $961(39.79)$ & $675(39.94)$ & $286(39.45)$ & \\
\hline Yes & $1454(60.21)$ & $1015(60.06)$ & $439(60.55)$ & \\
\hline Mechanical ventilation, $n(\%)$ & & & & 0.140 \\
\hline No & $1138(47.12)$ & $787(46.57)$ & $351(48.41)$ & \\
\hline Yes & $1277(52.88)$ & $903(53.43)$ & $374(51.59)$ & \\
\hline \multicolumn{5}{|l|}{ Scoring systems } \\
\hline APS III & $53(41-68)$ & $53(41-67)$ & $53(41-68)$ & 0.830 \\
\hline SAPS II & $41(32-50)$ & $40(31-50)$ & $41(32.50-51)$ & 0.830 \\
\hline \multicolumn{5}{|l|}{ Vital signs } \\
\hline Heart rate (beats/minute) & $91(80-104)$ & $92(80-104)$ & $91(80-103)$ & 0.200 \\
\hline Systolic pressure (mmHg) & $107(100-114)$ & $107(100-115)$ & $107(100-114)$ & 0.490 \\
\hline Diastolic pressure $(\mathrm{mmHg})$ & $57(52-63)$ & $57(51-63)$ & $57(52-63)$ & 0.710 \\
\hline Respiratory rate (beats/minute) & $20(17-24)$ & $20(17-24)$ & $20(17-24)$ & 0.630 \\
\hline
\end{tabular}


TABLE 1: Continued.

\begin{tabular}{|c|c|c|c|c|}
\hline Characteristics & Total $(n=2415)$ & Train cohort $(\mathrm{n}=1690)$ & Validation cohort $(\mathrm{n}=725)$ & $P$ value \\
\hline Temperature $\left({ }^{\circ} \mathrm{C}\right)$ & $36.8(36.4-37.3)$ & $36.8(36.4-37.3)$ & $36.8(36.4-37.3)$ & 0.370 \\
\hline $\mathrm{SpO} 2(\%)$ & 97.4(95.9-98.7) & $97.4(95.9-98.6)$ & $97.5(95.9-98.8)$ & 0.140 \\
\hline \multicolumn{5}{|l|}{ Laboratory test } \\
\hline Anion gap $(\mathrm{mmol} / \mathrm{l})$ & $14.0(12.5-16.5)$ & $14.0(12.0-16.5)$ & $14.0(12.5-16.3)$ & 0.950 \\
\hline Bicarbonate $(\mathrm{mEq} / \mathrm{l})$ & $22.0(19-25.5)$ & $22.0(19.0-25.5)$ & $22.0(18.5-25.5)$ & 0.150 \\
\hline Bilirubin (mg/dl) & $0.6(0.4-1.3)$ & $0.6(0.4-1.3)$ & $0.6(0.4-1.3)$ & 0.180 \\
\hline Creatinine $(\mathrm{mg} / \mathrm{dl})$ & $1.1(0.8-1.6)$ & $1.1(0.8-1.6)$ & $1.1(0.8-1.6)$ & 0.740 \\
\hline Chloride (mEq/l) & $105.5(101.5-109.5)$ & $105.5(101.5-109.5)$ & $106.0(101.5-110.0)$ & 0.150 \\
\hline Glucose (mg/dl) & $134.5(109.5-171)$ & $134.5(110.0-170.13)$ & $135.5(108.5-172.8)$ & 0.790 \\
\hline Lactate $(\mathrm{mmol} / \mathrm{l})$ & $30.9(27.9-34.4)$ & $1.9(1.4-2.9)$ & $2.0(1.4-3.1)$ & 0.290 \\
\hline Platelets (K/UL) & $217.5(137-305)$ & $215.0(135.5-305.0)$ & $224.50(143.5-306.8)$ & 0.300 \\
\hline Potassium (mEq/l) & $4.1(3.8-4.6)$ & $4.1(3.8-4.6)$ & $4.1(3.8-4.6)$ & 0.870 \\
\hline PTT (seconds) & $34.6(29.2-43.5)$ & $34.7(29.3-43.7)$ & $34.3(28.9-42.93)$ & 0.210 \\
\hline APTT (seconds) & $15.3(13.6-18.35)$ & $15.3(13.7-18.5)$ & $15.1(13.5-18.2)$ & 0.150 \\
\hline BUN (mg/dl) & $24(15.5-38.5)$ & $24.0(15.5-39.0)$ & $24.5(16.0-37.5)$ & 0.460 \\
\hline WBC (K/UL) & $12.7(8.1-17.9)$ & $12.7(8.0-17.9)$ & $12.70(8.5-18.0)$ & 0.310 \\
\hline Neutrophils (\%) & $78.9(65.7-87)$ & $78.7(65.0-87.0)$ & $79.5(67.0-87.0)$ & 0.460 \\
\hline Lymphocytes (\%) & $9(5-15.4)$ & $9.0(5.0-15.4)$ & $9.0(5.0-15.5)$ & 0.960 \\
\hline \multicolumn{5}{|l|}{ Culture } \\
\hline Gram-positive bacteria, $n(\%)$ & & & & 0.300 \\
\hline No & $1914(79.25)$ & $1344(79.53)$ & $570(78.62)$ & \\
\hline Yes & $501(20.75)$ & $346(20.47)$ & $155(21.38)$ & \\
\hline Gram-negative bacteria, $n(\%)$ & & & & 0.490 \\
\hline No & $2122(87.87)$ & $1489(88.11)$ & $633(87.31)$ & \\
\hline Yes & $293(12.13)$ & $201(11.89)$ & $92(12.69)$ & \\
\hline AKI & & & & 0.650 \\
\hline No & $849(35.16)$ & $599(35.44)$ & $250(34.48)$ & \\
\hline Yes & $1566(64.84)$ & $1091(64.56)$ & $475(65.52)$ & \\
\hline
\end{tabular}

NSAIDs: nonsteroidal anti-inflammatory drugs; ACEI: angiotensin-converting enzyme inhibitors; ARBs: angiotensin receptor blockers; APS III: acute physiology score III; SAPS II: simplified acute physiology score II; PTT: prothrombin time; APTT: activated partial thromboplastin time; BUN: blood urea nitrogen; WBC: white blood cell; AKI: acute kidney injury.

or chronic kidney disease upon admission. For patients older than 89 years, the actual age could not be obtained due to the date of birth being 300 years before the first admission [19]. Thus, our study excluded age over 89 years old.

2.3. Diagnosis of Septic Shock and AKI. According to the Third International Consensus Definitions (ICDs) for Sepsis and Septic Shock (Septic-3), septic shock was defined as an infection or a suspected infection with a vasopressor requirement to maintain a mean arterial pressure of $65 \mathrm{mmHg}$ or greater and serum lactate level of $>2 \mathrm{mmol} / \mathrm{l}(18 \mathrm{mg} / \mathrm{dl})$ in the absence of hypovolemia [20]. In the present study, we identified patients suffering from septic shock based on the ICD-9 (78552). The primary outcome of this study was the development of AKI within $48 \mathrm{~h}$ after ICU admission. A diagnosis of AKI was made according to the acute kidney injury network (AKIN) criteria [21], including the increase of serum creatinine $\geq 26.5 \mu \mathrm{mol} / \mathrm{l}$ or 1.5 times higher than baseline within $48 \mathrm{~h}$ or urine out $<0.5 \mathrm{ml} /(\mathrm{kg} \cdot \mathrm{h})$ for more than $6 \mathrm{~h}$.
The reasons for the use of AKIN rather than KDIGO criteria for AKI diagnosis in this study are as follows: (1) the data recorded by the MIMIC-III database were earlier than the release time of KDIGO criteria and (2) KDIGO criteria were based on the changes of kidney function within 7 days, but many confounding factors, such as antibiotics and nosocomial infection, may affect renal function [22].

2.4. Research Variables. Data of each patient, including demographic characteristics, comorbidity, vital signs, interventions, and laboratory examinations, were obtained from the MIMIC-III database [23, 24]. Demographic characteristics, including age, gender, and race, were collected from the original database. Vital signs, including respiratory rate (beats/min), heart rate body (beats/min), and temperature $\left({ }^{\circ} \mathrm{C}\right)$ upon ICU admission, were collected from charting in CHARTENS. Laboratory parameters, including WBC count, hemoglobin, platelet count, BUN, potassium, bicarbonate, chloride, neutrophils, lymphocytes, and lactic acid, were 
TABLE 2: Results of the multicollinearity diagnosis.

\begin{tabular}{|c|c|}
\hline Variables & Variance expansion factor \\
\hline Gender & 1.13 \\
\hline Age & 1.99 \\
\hline Ethnicity & 1.10 \\
\hline Congestive heart failure & 1.29 \\
\hline Hypertension & 1.21 \\
\hline Diabetes & 1.26 \\
\hline Obesity & 1.13 \\
\hline APSIII & 1.04 \\
\hline SAPS II & 3.20 \\
\hline Aminoglycoside & 1.08 \\
\hline Glycopeptide antibiotics & 1.20 \\
\hline NSAIDs & 1.17 \\
\hline Stain & 1.17 \\
\hline ACEI/ARBs & 1.27 \\
\hline Heart rate & 1.55 \\
\hline Systolic pressure & 1.68 \\
\hline Diastolic pressure & 1.79 \\
\hline Respiratory rate & 1.33 \\
\hline Temperature & 1.31 \\
\hline $\mathrm{SpO} 2$ & 1.22 \\
\hline Anion gap & 3.18 \\
\hline Bicarbonate & 3.05 \\
\hline Bilirubin & 1.29 \\
\hline Creatinine & 2.37 \\
\hline Chloride & 1.95 \\
\hline Glucose & 1.29 \\
\hline Lactate & 1.63 \\
\hline Platelets & 1.54 \\
\hline Potassium & 1.23 \\
\hline PTT & 1.15 \\
\hline APTT & 1.17 \\
\hline BUN & 2.23 \\
\hline WBC & 1.19 \\
\hline Neutrophils & 1.03 \\
\hline Lymphocytes & 1.10 \\
\hline Gram-positive bacteria & 1.08 \\
\hline Gram-negative bacteria & 1.12 \\
\hline Mechanical ventilation & 1.60 \\
\hline
\end{tabular}

NSAIDs: nonsteroidal anti-inflammatory drugs; ACEI: angiotensinconverting enzyme inhibitors; ARBs: angiotensin receptor blockers; APS III: acute physiology score III; SAPS II: simplified acute physiology score II; SBP: systolic blood pressure; DBP: diastolic blood pressure; PTT: prothrombin time; APTT: activated partial thromboplastin time; BUN: blood urea nitrogen; WBC: white blood cell; AKI: acute kidney injury.

recorded in the table of laboratory events. Interventions, including mechanical ventilation, angiotensin-converting enzyme inhibitors (ACEIs), and angiotensin receptor blockers (ARBs), were recorded in the first $24 \mathrm{~h}$ after admission. Acute physiology score III (APS III) and Simplified Acute Physiology Score (SAPS II) were calculated using the
TABLE 3: Univariate logistic regression analysis of predictive variables of AKI in the training cohort.

\begin{tabular}{|c|c|c|c|}
\hline Variables & OR & $95 \% \mathrm{CI}$ & $P$ value \\
\hline \multicolumn{4}{|l|}{ Demographic variables } \\
\hline Age (years) & 1.01 & $1.17-1.89$ & 0.340 \\
\hline Female, $n(\%)$ & 1.49 & $0.99-1.02$ & $<0.001$ \\
\hline Non-Caucasian, $n(\%)$ & 0.59 & $0.45-0.77$ & $<0.001$ \\
\hline Obesity, $n(\%)$ & 2.61 & $1.02-1.76$ & $<0.001$ \\
\hline \multicolumn{4}{|l|}{ Comorbidities, $n(\%)$} \\
\hline Congestive heart failure, $n(\%)$ & 1.34 & $0.95-1.57$ & 0.030 \\
\hline Hypertension, $n(\%)$ & 1.22 & $1.04-1.81$ & 0.130 \\
\hline Diabetes, $n(\%)$ & 1.38 & $1.43-4.78$ & 0.020 \\
\hline \multicolumn{4}{|l|}{ Interventions } \\
\hline Aminoglycoside, $n(\%)$ & 0.99 & $1.00-1.01$ & 0.960 \\
\hline Glycopeptide antibiotics, $n(\%)$ & 0.81 & $1.03-1.06$ & 0.130 \\
\hline NSAIDs, $n(\%)$ & 0.96 & $0.97-1.08$ & 0.740 \\
\hline Stain, $n(\%)$ & 1.25 & $0.60-1.63$ & 0.180 \\
\hline ACEI/ARBs, $n(\%)$ & 1.72 & $0.62-1.06$ & $<0.001$ \\
\hline Mechanical ventilation, $n(\%)$ & 1.87 & $1.41-2.47$ & $<0.001$ \\
\hline \multicolumn{4}{|l|}{ Scoring systems } \\
\hline APSIII & 1.00 & $0.73-1.25$ & 0.560 \\
\hline SAPSII & 1.05 & $0.91-1.71$ & $<0.001$ \\
\hline Vital signs & 1.02 & $1.34-2.21$ & 0.410 \\
\hline Heart rate (beats/minute) & 1.01 & $1.00-1.02$ & 0.230 \\
\hline Systolic pressure (mmHg) & 1.00 & $0.98-1.01$ & 0.700 \\
\hline Diastolic pressure (mmHg) & 0.99 & $0.98-1.01$ & 0.420 \\
\hline Respiratory rate (beats/minute) & 1.00 & $0.97-1.03$ & 1.000 \\
\hline Temperature $\left({ }^{\circ} \mathrm{C}\right)$ & 0.85 & $0.71-1.02$ & 0.090 \\
\hline $\mathrm{SpO} 2(\%)$ & 0.96 & $0.91-1.02$ & 0.230 \\
\hline \multicolumn{4}{|l|}{ Laboratory test } \\
\hline Anion gap (mmol/l) & 1.00 & $0.94-1.06$ & 0.900 \\
\hline Bicarbonate (mEq/l) & 1.03 & $0.99-1.07$ & 0.130 \\
\hline Bilirubin (mg/dl) & 1.07 & $1.03-1.13$ & $<0.001$ \\
\hline Creatinine (mg/dl) & 1.25 & $1.05-1.48$ & 0.010 \\
\hline Chloride (mEq/l) & 0.99 & $0.96-1.01$ & 0.190 \\
\hline Glucose (mg/dl) & 1.00 & $0.99-1.00$ & 0.130 \\
\hline Lactate $(\mathrm{mmol} / \mathrm{l})$ & 1.07 & $0.96-1.19$ & 0.230 \\
\hline Platelets (K/Ul) & 1.00 & $0.99-1.01$ & 0.730 \\
\hline Potassium (mEq/l) & 1.04 & $0.85-1.27$ & 0.700 \\
\hline PTT (seconds) & 1.00 & $0.99-1.01$ & 0.230 \\
\hline APTT (seconds) & 0.99 & $0.98-1.00$ & 0.050 \\
\hline BUN (mg/dl) & 0.99 & $0.98-1.00$ & $<0.001$ \\
\hline WBC (K/Ul) & 0.99 & $0.98-1.01$ & 0.240 \\
\hline Neutrophils (\%) & 1.00 & $0.99-1.01$ & 0.930 \\
\hline Lymphocytes (\%) & 1.00 & $0.99-1.01$ & 0.310 \\
\hline \multicolumn{4}{|l|}{ Culture } \\
\hline Gram positive bacteria, $n(\%)$ & 1.35 & $1.01-1.81$ & 0.050 \\
\hline Gram negative bacteria, $n(\%)$ & 1.04 & $0.71-1.52$ & 0.850 \\
\hline
\end{tabular}

CI: confidence interval; OR: odds ratio; NSAIDs: nonsteroidal antiinflammatory drugs; ACEI: angiotensin-converting enzyme inhibitors; ARBs: angiotensin receptor blockers; APS III: acute physiology score III; SAPS II: simplified acute physiology score II; PTT: prothrombin time; APTT: activated partial thromboplastin time; BUN: blood urea nitrogen; WBC: white blood cell; AKI: acute kidney injury. 
TABLE 4: Multivariate logistic regression analysis of risk factors of AKI in the training cohort.

\begin{tabular}{|c|c|c|c|}
\hline Variables & OR & $95 \% \mathrm{CI}$ & $P$ value \\
\hline Gender (female vs. male) & 1.41 & $1.13-1.77$ & $<0.001$ \\
\hline $\begin{array}{l}\text { Ethnicity } \\
\text { (non-Caucasian vs. Caucasian) }\end{array}$ & 0.61 & $0.47-0.78$ & $<0.001$ \\
\hline $\begin{array}{l}\text { Congestive heart failure } \\
\text { (yes vs. no) }\end{array}$ & 1.53 & $1.19-1.97$ & $<0.001$ \\
\hline Diabetes (yes vs. no) & 1.32 & $1.03-1.68$ & 0.030 \\
\hline Obesity (yes vs. no) & 2.98 & $1.66-5.34$ & $<0.001$ \\
\hline SAPS II & 1.05 & $1.04-1.06$ & $<0.001$ \\
\hline ACEI/ARBs (yes vs. no) & 1.78 & $1.40-2.25$ & $<0.001$ \\
\hline Bilirubin (mg/dl) & 1.08 & $1.03-1.12$ & $<0.001$ \\
\hline Creatinine (mg/dl) & 1.20 & $1.04-1.38$ & 0.010 \\
\hline BUN (mg/dl) & 0.99 & $0.98-0.99$ & $<0.001$ \\
\hline Mechanical ventilation (yes vs. no) & 1.69 & $1.33-2.15$ & $<0.001$ \\
\hline Constant & 0.12 & & $<0.001$ \\
\hline
\end{tabular}

OR: odds ratio; CI: confidence interval; ACEI: angiotensin-converting enzyme inhibitors; ARB: angiotensin receptor blockers; SAPS II: simplified acute physiology score II; BUN: blood urea nitrogen; AKI: acute kidney injury.

data of the first $48 \mathrm{~h}$ of the ICU stay. All comorbidities were identified according to the ICD-9 code records. Variables with $>20 \%$ missing values were excluded from further analysis, and variables with $\leq 20 \%$ missing values were filled with the multiple imputation.

2.5. Statistical Analysis. All eligible patients were divided into the training and validation cohorts with the split ratio of $7: 3$. The data of the training cohort were used to perform the logistic regression analysis and construct the nomogram, whereas the data in the validation cohort were used to validate the model. Continuous variables were expressed as median with quartile. These data were compared by $t$-test or rank sum test, as appropriate. $\chi^{2}$ test or Fisher's exact test was used to compare the categorical variables. Potential multicollinearity between variables was judged by the variance expansion factor (VIF). A VIF of $\geq 5$ was considered evidence of multicollinearity [25]. Univariate and multivariate logistic regression models were used to select the independent risk factors of AKI in the training cohort. According to the results of multivariate logistic regression analysis, the nomogram was developed to predict the risk of AKI in patients suffering from septic shock in the ICU. The performance of the nomogram was first quantified in the training cohort and then in the validation cohort in terms of discrimination, calibration, and clinical utility. The discrimination of the nomogram was evaluated by the area under curve (AUC) of the receiver operating characteristics (ROC) curve. The calibration curve was drawn to examine the consistence of the predicted probabilities and the observed outcomes. The clinical applicability of the nomogram was estimated by the decision curve analysis (DCA) and clinical impact curve. The statistical significance of all analyses was set at a $P$ level less than 0.05 . All analyses were performed using $\mathrm{R}$ version 4.0.5 (http://www.r-project.org/).

\section{Results}

3.1. Baseline Characteristics. The procedure for subject selection is shown in Figure 1. A total of 2415 patients suffering from septic shock were included in the final analysis. Among these, 1566 patients (64.84\%) developed AKI in $48 \mathrm{~h}$ after ICU admission. Thirty-eight variables, namely, age, gender, ethnicity, obesity, congestive heart failure, hypertension, diabetes, aminoglycoside, glycopeptide antibiotics, nonsteroidal antiinflammatory drugs (NSAIDs), stain, ACEI/ARBs, APSIII, SAPSII, heart rate, systolic pressure, diastolic pressure, respiratory rate, temperature, $\mathrm{SpO}$, anion gap, bicarbonate, bilirubin, creatinine, chloride, glucose, lactate, platelets, potassium, prothrombin time (PTT), activated partial thromboplastin time (APTT), BUN, white blood cell (WBC), neutrophils, lymphocytes, Gram-positive bacteria, Gram-negative bacteria, and mechanical ventilation, were collected from the MIMIC-III database. The clinicopathological characteristics of the eligible patients are shown in Table 1. The baseline clinicopathologic data were similar between the training and testing sets. AKI was detected in $64.48 \%(1091 / 1690)$ and $65.52 \%(475 / 725)$ of the patients in the training and validation sets, respectively.

3.2. Predictors of AKI and Nomogram Development. After multicollinearity examination, no multicollinearity was found between variables, because all VIFs were less than 5 (Table 2). Thus, all 38 features were included in the univariate logistic regression analysis. Based on the univariate logistic regression analyses, 11 variables, namely, gender, race, congestive heart failure, diabetes, obesity, SAPS II, ACEI/ARBs, bilirubin, creatinine, BUN, and mechanical ventilation, were significantly associated with AKI development in patients suffering from septic shock (Table 3). In multivariate analysis, gender, race, congestive heart failure, diabetes, obesity, SAPS II, ACEI/ARBs, bilirubin, creatinine, $B U N$, and mechanical ventilation were identified as the independent risk factors for AKI in patients suffering from septic shock $(P<0.05$, Table 4$)$. Therefore, a nomogram for predicting AKI in patients suffering from septic shock was constructed based on these variables (Figure 2).

3.3. Nomogram Validation. First, the ROC curves are shown in Figure 3, and the discriminant results showed that the model has a good ability to distinguish AKI patients from non-AKI patients with AUCs of 0.756 and 0.760 in the training and validation cohorts, respectively. Second, the predictive probabilities of the model were in consistent agreement with the observation results in both the training and validation sets, thereby suggesting a good calibration (Figure 4). Third, the DCA (Figure 5) and clinical impact curve analysis (Figure 6) visually showed that the nomogram had superior overall net benefit within the wide and practical ranges of threshold probabilities and impacted patient outcomes, thereby indicating that the nomogram had significant predictive value. When the predicted probability thresholds were set as 30\%-100\% and 30\%-93\% in the training and validation cohorts, the net benefit ranges were $0 \%-50 \%$ and $0 \%-47 \%$, respectively. The smaller the threshold was, the better the net benefit was. 
Points

Sex

Ethnicity

Congestive_heart_failure

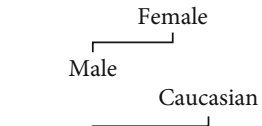

Non-caucasian

Diabetes

Obesity
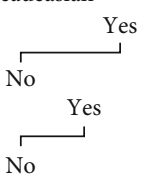

SAPSII

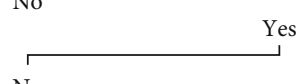

No

SAPSII

ACEI/ARBs

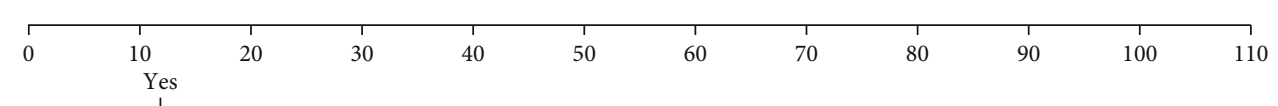

No

Bilirubin

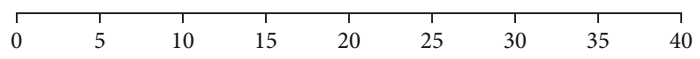

Creatinine

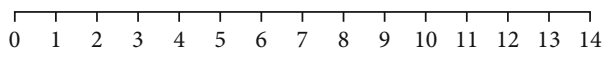

BUN

Mechanical_ventilation

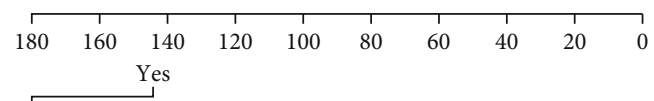

No

Total points

\begin{tabular}{rrrrrrrrrr}
\hline \\
0 & 40 & 60 & 80 & 100 & 120 & 140 & 160 & 180 & 200
\end{tabular}

AKI

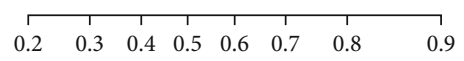

FIGURE 2: Nomogram to identify the risk of AKI in septic shock, based on logistic regression analysis. To acquire the corresponding scores for each variable, draw a vertical line upward to the "Points" axis. Sum the score for all predictors and locate the final value on the "Total Points" axis. Draw a line straight down to the "Probability of AKI" axis to determine the risk of AKI. Abbreviations: AKI: acute kidney injury; SAPS II: Simplified Acute Physiology Score II; ACEI: angiotensin-converting enzyme inhibitors; ARBs: angiotensin receptor blockers; BUN: blood urea nitrogen.

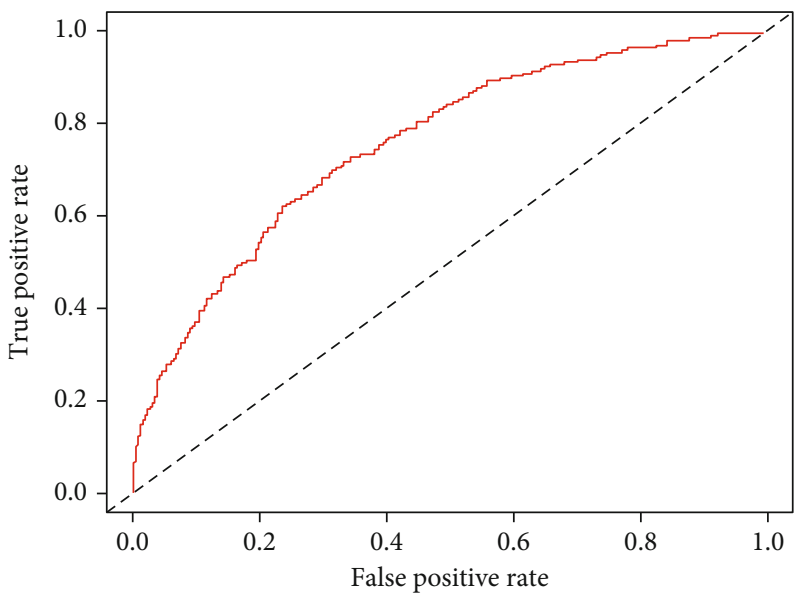

(a)

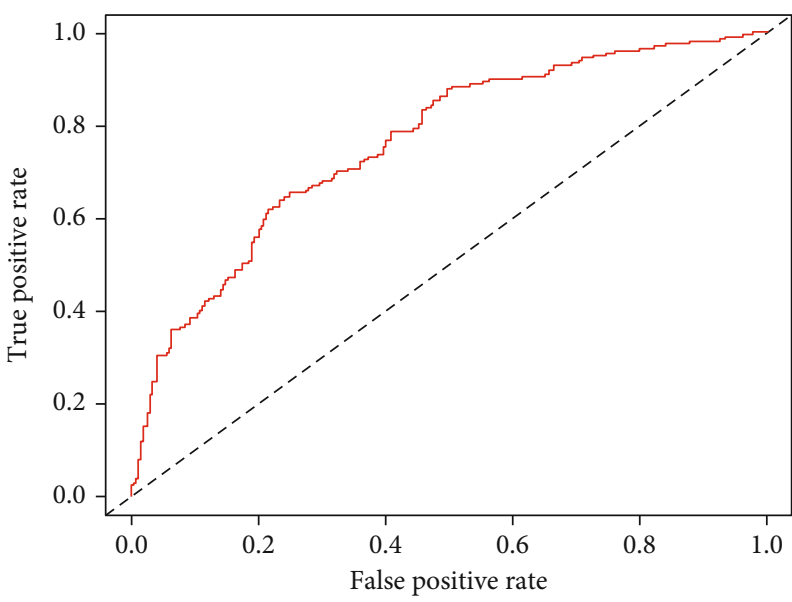

(b)

FIGURE 3: Receiver operating characteristic curve of the nomogram. Receiver operating characteristic curve for predicting AKI in septic shock patients during the intensive care admission. AUC: area under the receiver operating characteristic curve. The AUC of the nomogram for the prediction of AKI in septic shock patients was 0.756 in the training set and 0.760 in the validation set. 
Calibration curve

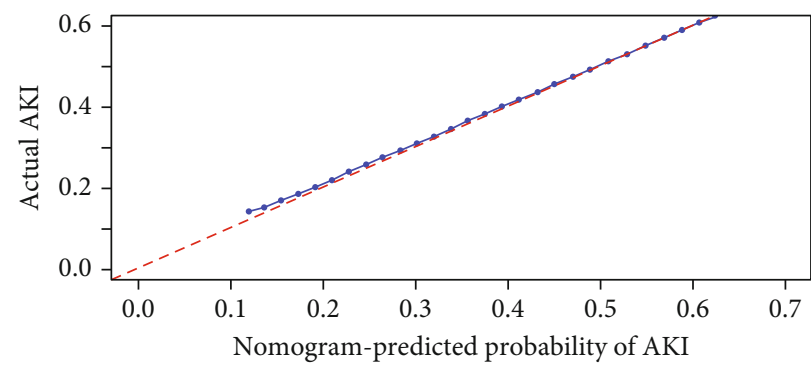

(a)

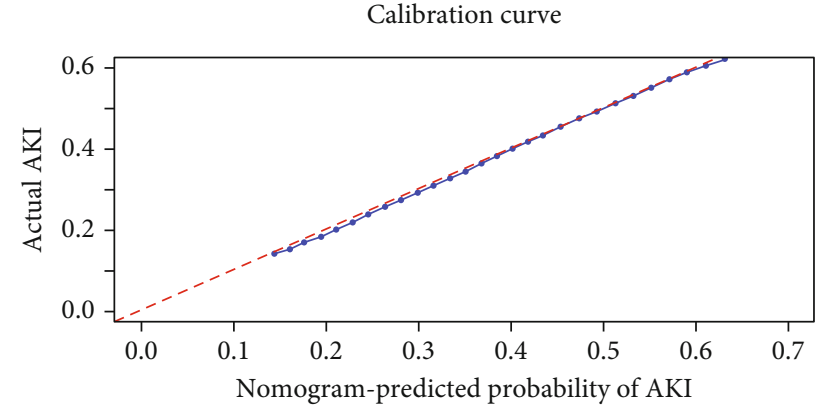

(b)

FIgURE 4: Calibration curves of the predicted nomogram in the training set (a) and validation set (b). The $x$-axis represents the predicted probability calculated by the nomogram, and the $y$-axis is the observed actual probability of AKI. The clinodiagonal represents a perfect prediction by an ideal model. The solid curve represents the initial cohort and the dotted curve is bias corrected by bootstrapping ( $B=1000$ repetitions), which demonstrates the performance of the predicted model.

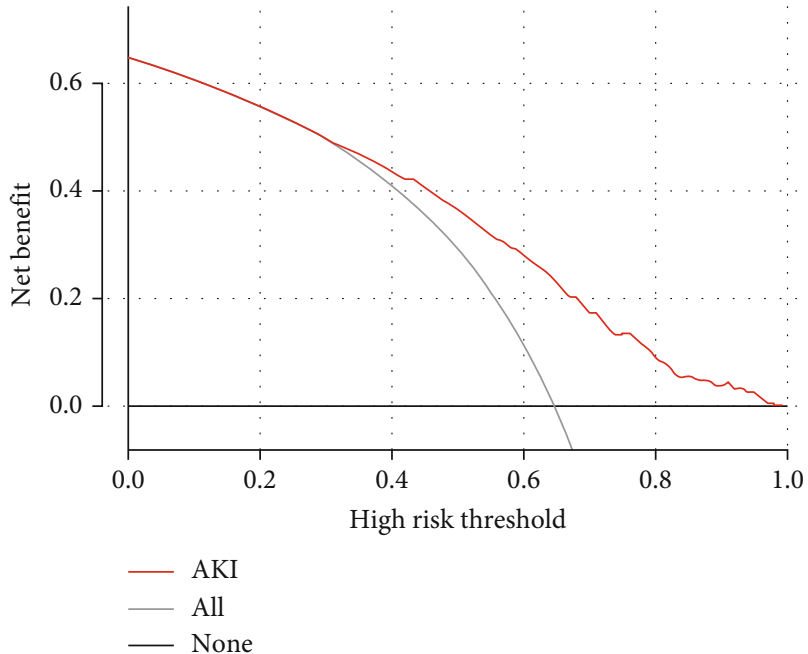

(a)

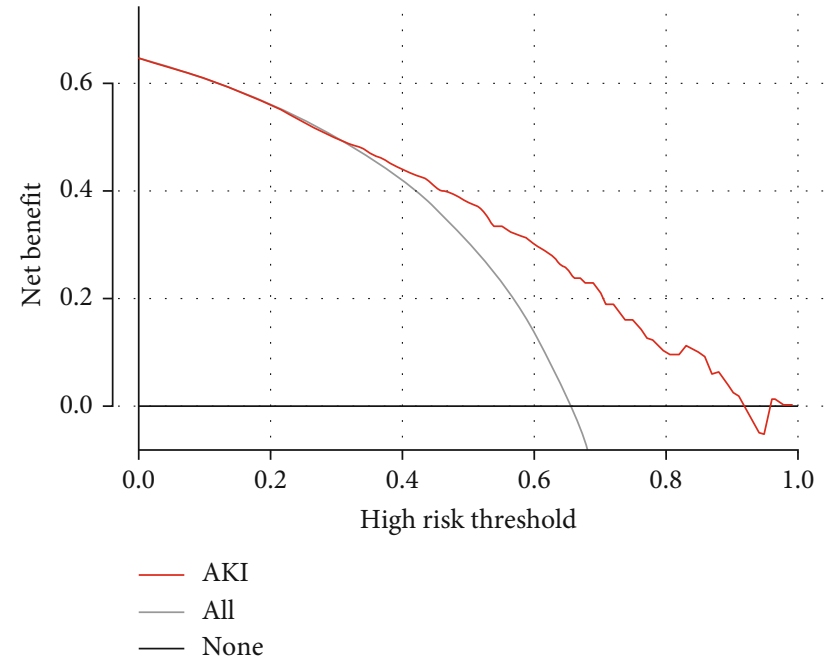

(b)

FIGURE 5: Decision curve analysis (DCA) of the nomogram in the training set (a) and the validation set (b). The horizontal line indicates no patients develop acute kidney injury (AKI), and the gray oblique line indicates patients develop AKI. The red solid line represents the AKI risk nomogram. In DCA, the nomogram shows a more net benefit than full or no treatment across a threshold probability range. DCA: decision curve analysis; AKI: acute kidney injury.

\section{Discussion}

The incidence of AKI in septic shock patients reached as high as $64.5 \%$ in the ICU, similar to the findings of previously published studies. According to a report in Finland, a total of 488 (53.2\%) AKI incidences were detected among 918 patients with severe sepsis during their stay in the ICU [26]. Another study found that AKI developed in 572 (57.7\%) of the 992 patients with sepsis and septic shock patients [15]. However, the incidence rate of AKI was higher in patients suffering from septic shock than in patients suffering from other diseases. For example, the incidence of AKI was $40 \%-50 \%$ among patients with sepsis in the ICU [5]. Fan et al. reported that AKI was developed in $41.3 \%$ (314/760) of the patients with diabetic ketoacidosis after ICU admission [17]. Several mechanisms might contribute to the pathogenesis of AKI in sepsis, including inflamma- tion, microcirculatory dysfunction, and mitochondrial dysfunction [5]. The kidney is the most common organ involved in sepsis, and septic patients with kidney dysfunction have a higher risk of death than those without kidney dysfunction $[27,28]$. In sepsis, circulating toxins act on the vascular endothelium, reducing microcirculation blood flow and producing a large number of inflammatory mediators, such as tumor necrosis factor and transforming growth factor $\beta$. Inflammatory response waterfall can lead to "intrarenal shunting," in which the renal blood flow is shunted from the medulla to the cortex, resulting in medulla hypoperfusion even with increased renal blood flow [29]. Simultaneously, the tissue damage caused by sepsis increases the inducible nitric oxide (NO) synthase and decreases endothelial NO synthase activity. The dysfunction of NO synthase decreases nitricoxide-mediated endothelium-dependent vasodilation, resulting in the imbalance of local renal 


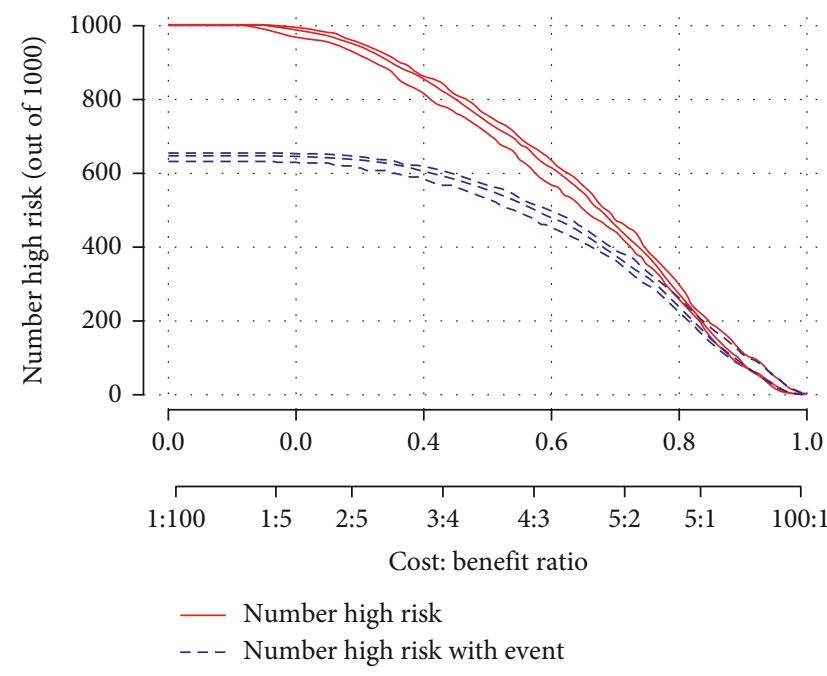

(a)

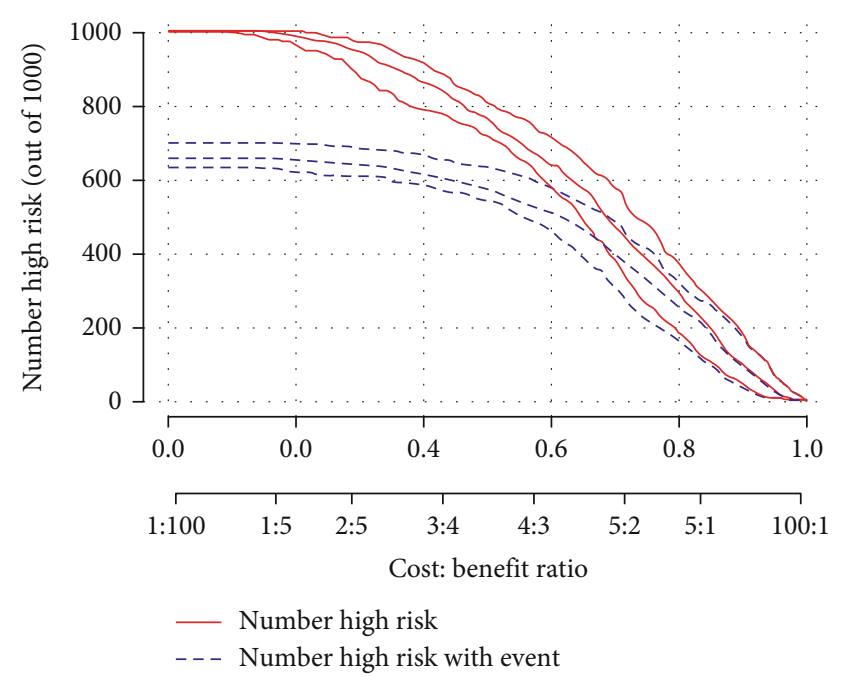

(b)

Figure 6: Clinical impact curve (CIC) of nomogram. The red curve (number of high-risk individuals) indicates the number of people who are classified as positive (high risk) by the model at each threshold probability; the blue curve (number of high-risk individuals with outcome) is the number of true positives at each threshold probability. CIC visually indicated that nomogram conferred high clinical net benefit and confirmed the clinical value.

microcirculation [30]. Moreover, in sepsis, the activation of bacterial phospholipids, related cytokines, and chemical factors in the circulatory system are involved in the occurrence of tubular epithelial cell damage, leading to renal tubular necrosis, which is an important cause of AKI [29]. In addition, under the stress state caused by sepsis, mitochondrial autophagy increases to remove damaged mitochondria, thus playing a role in protecting the kidney. However, with the progress of the disease, mitochondrial autophagy is inhibited, leading to the accumulation of damaged mitochondria, thereby mediating kidney damage [31].

Logistic regression analysis showed that gender, race, congestive heart failure, diabetes, obesity, SAPS II, ACEI/ ARBs, bilirubin, creatinine, BUN, and mechanical ventilation were significantly associated with AKI risk in septic shock. Compared with previous studies on sepsis-related AKI, this study had some new findings. Firstly, SAPS II score was the most sensitive indicator for predicting the risk of AKI in septic shock. SAPS II score is among the most commonly used methods to quantify the death risk of AKI patients in ICU. The higher the SAPS II score was, the greater the risk of AKI was. When patients develop AKI after ICU admission, SAPS II showed the best performance in the prediction of AKI among all scoring systems, including Acute Physiology and Chronic Health Evaluation II (APACHE II), Sequential Organ Failure Assessment (SOFA), Logistic Organ Dysfunction Score, and Organ System Failure [32]. SAPS II score was superior to APACHE II and SOFA scores, because it can predict the survival outcome in patients suffering from septic shock [33]. Secondly, hyperbilirubinemia is a common complication in septic patients; the bilirubin level in septic shock patients significantly increases in $72 \mathrm{~h}$ after ICU admission [34]. Hyperbilirubinemia can be found in $60 \%$ of patients with AKI [35]. Elevated serum bilirubin levels are an independent risk factor for the occurrence of AKI [36]. Elevated bilirubin may produce oxidative stress on renal tubular cells, trigger apoptosis, and aggravate renal ischemia-reperfusion injury that contributes to the development of AKI [37, 38]. Thirdly, AKI is characterized by a sharp drop in the glomerular filtration rate and a rapid increase in serum creatinine, BUN, sodium, and water storage. Serum creatinine and BUN are common indicators of renal function dysfunction. Studies have shown that even slightly elevated levels of serum creatinine and BUN are significantly associated with an increasing risk of AKI after ICU admission [39]. Moreover, modest changes of serum creatinine were significantly associated with higher mortality, longer length of hospital stay, and heavier costs in patients with AKI [39].

Other risk factors were independently associated with AKI risk, including gender, complicating diseases (diabetes, congestive heart failure, and obesity), mechanical ventilation, and ACEI/ARBs therapy. Women were more prone to develop AKI due to the effect of estradiol level on patients suffering from septic shock. Ovarian levels of estradiol at $>40 \mathrm{pg} / \mathrm{ml}$ were an independent risk factor of septic shockrelated AKI in women [40]. In a matched case-control study, Kim et al. found that the incidence rate of postoperative AKI was significantly higher in patients with diabetes than those without diabetes [41]. Diabetes can increase the susceptibility of renal ischemia/reperfusion injury [42]. Congestive heart failure causes the complex interaction between the heart and kidney and results in the damage of renal function. Decrease in renal perfusion is the main factor responsible for development of AKI in patients with congestive heart failure [43]. Gameiro et al. proposed that the risk of AKI development was 2.31 times higher in obese patients with sepsis than in nonobese patients [44]. A complex interplay of different mechanisms may increase the susceptibility to AKI in critically ill obese patients, such as glomerulopathy, low 
inflammatory status, endothelial dysfunction, enhanced oxidative stress, activation of the renin-angiotensin-aldosterone system, and increased sympathetic nervous system activity [45]. Mechanical ventilation is connected with a threefold increase in the risk of developing AKI among critically ill patients [46]. Ventilator-induced lung injury might contribute to the development of AKI during the use of mechanical ventilation [47]. Moreover, the use of ACEI/ARBs can cause efferent arteriole dilatation, decrease renal blood flow, and reduce glomerular pressure, which may increase the risk of AKI in patients suffering from septic shock [48].

In this study, we developed a simple and rapid nomogram model for predicting AKI risk in patients suffering from septic shock. The variables identified by our nomogram can be easily obtained in clinic and can reflect the disease activity of patients, thereby providing clinically relevant information in the management of patients suffering from septic shock. The proposed nomogram showed a good performance in the discrimination, calibration, and clinical application and provided valuable information for the decision-making of the appropriated therapy options for individual patients. More importantly, it filled the gap between the high incidence of AKI in septic shock and the lack of reliable predictive model. We cited an example to show how to use the nomogram model. For example, we assumed that a Caucasian man with septic shock and diabetes had received mechanical ventilation with a SAPS II score of 45 , a BUN level of $80 \mathrm{mg} / \mathrm{dl}$, a bilirubin level of $15 \mathrm{mg} / \mathrm{dl}$, and a creatinine level of $5 \mathrm{mg} / \mathrm{dl}$. According to Figure 2, the score corresponding to each individual parameter was obtained from the first row (the "Point" axis). For this patient, the score of gender was 0 based on the male sex, and the score of diabetes was 6 based on the complication of diabetes. Finally, the overall score was calculated as the sum of points for all parameters $(0(\operatorname{sex})+10$ (Caucasian $)+0$ (congestive heart failure $)+6$ (diabetes) +0 (obesity) +42 (SAPS II) +0 (ACEI/ARBs $)+21$ (bilirubin) + 18 (creatinine) + 29 (BUN) + 11 (mechanical ventilation $=137)$. This score corresponded to a risk of developing AKI at an approximately $87 \%$ level.

This study had several limitations. First, selection bias might be inevitable, because this study was a retrospective analysis of secondary data. Second, missing data were handled with multiple imputations, which might decrease the accuracy of the model. Third, the model was constructed based on a US population. Thus, its generalizability to the global population was still unclear. Fourth, the nomogram was developed and validated by the same database. Thus, it was more reliable to validate it prospectively or at least in another database. Fifth, because the nomogram was built based on 11 indicators, the sensitivity of the model performance may decline if the data of one or two indicators for a patient were missing. Finally, we only considered traditional parameters and did not consider some valuable biomarkers that might contribute to AKI development.

\section{Conclusion}

By incorporating 11 independent risk factors of AKI in septic shock, a simplified score model was constructed for AKI risk estimation in patients suffering from septic shock in the ICU. The proposed nomogram showed good performance in terms of discrimination, calibration, and clinical application. By early assessment of the risk of AKI development, clinicians can implement more measures that are beneficial for patients suffering from septic shock. Further studies are needed to externally validate our model using a largesample prospective cohort study.

\section{Data Availability}

The data support the findings of this study and are available from the corresponding author upon reasonable request.

\section{Ethical Approval}

Ethical approval was waived.

\section{Consent}

Informed consent was unnecessary because the MIMIC-III research data are anonymous and publicly available.

\section{Disclosure}

The opinions expressed in this study are those of the authors and do not represent the opinions of the Beth Israel Deaconess Medical Center.

\section{Conflicts of Interest}

All authors declare no conflict of interest.

\section{Authors' Contributions}

Concept and design were done by WJY, YSR, and LSS. Acquisition, analysis, or interpretation of data was performed by HXY, LJ, HXF, and WYF. Drafting of the manuscript was conducted by WJY, LSS, and HXF. Critical revision of the manuscript for important intellectual content was done by WJY. Statistical analysis was performed by HXF, HXY, LJ, and WYF. Supervision was done by WYF, LSS, and HXY. YSR had full access to all of the data in the study and takes responsibility for the integrity of the data and the accuracy of the data analysis. All authors read and approved the final manuscript. Suru Yue and Shasha Li contributed equally to this work.

\section{Acknowledgments}

The authors would like to thank MIMIC-III for open access to their database. This project was funded by the Guangdong Basic and Applied Basic Research Foundation (2018A0303130269 and 2020B1515020004), Guangdong Province Medical Scientific Research Fund Project (A2019537), Competitive Project of Financial Special Funds for Science and Technology of Zhanjiang (2018A01026), Guangdong Medical University Scientific Research Fund Project (GDMUM201806), and Affiliated Hospital of Guangdong Medical University Clinical Research Program (LCYJ2019B007 and LCYJ2018C004). 


\section{References}

[1] J. L. Vincent, G. Jones, S. David, E. Olariu, and K. K. Cadwell, "Frequency and mortality of septic shock in Europe and North America: a systematic review and meta-analysis," Critical Care, vol. 23, no. 1, p. 196, 2019.

[2] SepNet Critical Care Trials Group, "Incidence of severe sepsis and septic shock in German intensive care units: the prospective, multicentre INSEP study," European Journal of Intensive Care Medicine, vol. 42, no. 12, pp. 1980-1989, 2016.

[3] J. P. Quenot, C. Binquet, F. Kara et al., "The epidemiology of septic shock in French intensive care units: the prospective multicenter cohort EPISS study," Critical Care, vol. 17, no. 2, article R65, 2013.

[4] J. Liu, H. Xie, Z. Ye, F. Li, and L. Wang, "Rates, predictors, and mortality of sepsis-associated acute kidney injury: a systematic review and meta-analysis," BMC Nephrology, vol. 21, no. 1, p. $318,2020$.

[5] S. Peerapornratana, C. L. Manrique-Caballero, H. Gómez, and J. A. Kellum, "Acute kidney injury from sepsis: current concepts, epidemiology, pathophysiology, prevention and treatment," Kidney International, vol. 96, no. 5, pp. 1083-1099, 2019.

[6] F. S. de Oliveira, F. G. Freitas, E. M. Ferreira et al., "Positive fluid balance as a prognostic factor for mortality and acute kidney injury in severe sepsis and septic shock," Journal of Critical Care, vol. 30, no. 1, pp. 97-101, 2015.

[7] D. Y. Zhi, J. Lin, H. Z. Zhuang et al., "Acute kidney injury in critically ill patients with sepsis: clinical characteristics and outcomes," Journal of Investigative Surgery, vol. 32, no. 8, pp. 689-696, 2019.

[8] J. Xie, H. Wang, Y. Kang et al., "The epidemiology of sepsis in Chinese ICUs," Critical Care Medicine, vol. 48, no. 3, pp. e209e218, 2020.

[9] S. Coelho, G. Cabral, J. A. Lopes, and A. Jacinto, "Renal regeneration after acute kidney injury," Nephrology (Carlton, Vic.), vol. 23, no. 9, pp. 805-814, 2018.

[10] X. Bu, L. Zhang, P. Chen, and X. Wu, "Relation of neutrophilto-lymphocyte ratio to acute kidney injury in patients with sepsis and septic shock: a retrospective study," International Immunopharmacology, vol. 70, pp. 372-377, 2019.

[11] J. J. Wang, N. H. Chi, T. M. Huang et al., "Urinary biomarkers predict advanced acute kidney injury after cardiovascular surgery," Critical Care, vol. 22, no. 1, p. 108, 2018.

[12] M. Fiorentino, Z. Xu, A. Smith et al., "Serial measurement of cell-cycle arrest biomarkers [TIMP-2] · [IGFBP7] and risk for progression to death, Dialysis, or severe acute kidney injury in patients with septic shock.," American Journal of Respiratory and Critical Care Medicine, vol. 202, no. 9, pp. 12621270, 2020.

[13] Y. Xie, P. Huang, J. Zhang et al., "Biomarkers for the diagnosis of sepsis-associated acute kidney injury: systematic review and meta-analysis," Annals of Palliative Medicine, vol. 10, no. 4, pp. 4159-4173, 2021.

[14] S. Peerapornratana, P. Priyanka, S. Wang et al., "Sepsis-Associated Acute Kidney Disease," Kidney International Reports, vol. 5, no. 6, pp. 839-850, 2020.

[15] S. H. Suh, C. S. Kim, J. S. Choi, E. H. Bae, S. K. Ma, and S. W. Kim, "Acute kidney injury in patients with sepsis and septic shock: risk factors and clinical outcomes," Yonsei Medical Journal, vol. 54, no. 4, pp. 965-972, 2013.
[16] F. Deng, M. Peng, J. Li, Y. Chen, B. Zhang, and S. Zhao, "Nomogram to predict the risk of septic acute kidney injury in the first $24 \mathrm{~h}$ of admission: an analysis of intensive care unit data," Renal Failure, vol. 42, no. 1, pp. 428-436, 2020.

[17] T. Fan, H. Wang, J. Wang, W. Wang, H. Guan, and C. Zhang, "Nomogram to predict the risk of acute kidney injury in patients with diabetic ketoacidosis: an analysis of the MIMIC-III database," BMC Endocrine Disorders, vol. 21, no. 1, p. 37, 2021.

[18] A. E. Johnson, T. J. Pollard, L. Shen et al., "MIMIC-III, a freely accessible critical care database," Scientific Data, vol. 3, no. 1, article 160035, 2016.

[19] Y. Hu, Q. Cao, H. Wang et al., "Prognostic nutritional index predicts acute kidney injury and mortality of patients in the coronary care unit," Experimental and Therapeutic Medicine, vol. 21, no. 2, p. 123, 2021.

[20] M. Singer, C. S. Deutschman, C. W. Seymour et al., "The third international consensus definitions for sepsis and septic shock (Sepsis-3)," Journal of the American Medical Association, vol. 315, no. 8, pp. 801-810, 2016.

[21] M. E. Thomas, C. Blaine, A. Dawnay et al., "The definition of acute kidney injury and its use in practice," Kidney International, vol. 87, no. 1, pp. 62-73, 2015.

[22] A. E. W. Johnson, J. Aboab, J. D. Raffa et al., "A comparative analysis of sepsis identification methods in an electronic database," Critical Care Medicine, vol. 46, no. 4, pp. 494499, 2018.

[23] J. Yang, Y. Li, Q. Liu et al., "Brief introduction of medical database and data mining technology in big data era," Journal of Evidence-Based Medicine, vol. 13, no. 1, pp. 57-69, 2020.

[24] W. T. Wu, Y. J. Li, A. Z. Feng et al., "Data mining in clinical big data: the frequently used databases, steps, and methodological models," Military Medical Research, vol. 8, no. 1, p. 44, 2021.

[25] Y. Shen, X. Huang, and W. Zhang, "Platelet-to-lymphocyte ratio as a prognostic predictor of mortality for sepsis: interaction effect with disease severity-a retrospective study," BMJ Open, vol. 9, no. 1, article e022896, 2019.

[26] M. Poukkanen, S. T. Vaara, V. Pettilä et al., "Acute kidney injury in patients with severe sepsis in Finnish Intensive Care Units," Acta Anaesthesiologica Scandinavica, vol. 57, no. 7, pp. 863-872, 2013.

[27] J. T. Poston and J. L. Koyner, "Sepsis associated acute kidney injury,” BMJ, vol. 364, article k4891, 2019.

[28] A. Zarjou and A. Agarwal, "Sepsis and acute kidney injury," Journal of the American Society of Nephrology, vol. 22, no. 6, pp. 999-1006, 2011.

[29] F. Fani, G. Regolisti, M. Delsante et al., "Recent advances in the pathogenetic mechanisms of sepsis-associated acute kidney injury," Journal of Nephrology, vol. 31, no. 3, pp. 351-359, 2018.

[30] R. Bellomo, J. A. Kellum, C. Ronco et al., "Acute kidney injury in sepsis," Intensive Care Medicine, vol. 43, no. 6, pp. 816-828, 2017.

[31] J. Sun, J. Zhang, J. Tian et al., "Mitochondria in sepsis-induced AKI," Journal of the American Society of Nephrology, vol. 30, no. 7, pp. 1151-1161, 2019.

[32] V. T. Costa e Silva, I. de Castro, F. Liaño, A. Muriel, J. R. Rodríguez-Palomares, and L. Yu, "Sequential evaluation of prognostic models in the early diagnosis of acute kidney injury in the intensive care unit," Kidney International, vol. 75, no. 9, pp. 982-986, 2009. 
[33] A. M. Georgescu, J. Szederjesi, S. M. Copotoiu, and L. Azamfirei, "Predicting scores correlations in patients with septic shock - a cohort study," The Romanian Journal of Anaesthesia and Intensive Care, vol. 21, no. 2, pp. 95-98, 2014.

[34] J. J. Patel, A. Taneja, D. Niccum, G. Kumar, E. Jacobs, and R. Nanchal, "The association of serum bilirubin levels on the outcomes of severe sepsis," Journal of Intensive Care Medicine, vol. 30, no. 1, pp. 23-29, 2015.

[35] B. Suetrong, C. Pisitsak, J. H. Boyd, J. A. Russell, and K. R. Walley, "Hyperchloremia and moderate increase in serum chloride are associated with acute kidney injury in severe sepsis and septic shock patients," Critical Care, vol. 20, no. 1, p. 315, 2016.

[36] S. Mohammadi Kebar, S. Hosseini Nia, N. Maleki, A. Sharghi, and A. Sheshgelani, "The incidence rate, risk factors and clinical outcome of acute kidney injury in critical patients," Iranian Journal of Public Health, vol. 47, no. 11, pp. 1717-1724, 2018.

[37] L. Yuan, P. P. Liao, H. C. Song, J. H. Zhou, H. C. Chu, and L. Lyu, "Hyperbilirubinemia induces pro-apoptotic effects and aggravates renal ischemia reperfusion injury," Nephron, vol. 142, no. 1, pp. 40-50, 2019.

[38] C. Rafat, M. Burbach, I. Brochériou et al., "Bilirubin-associated acute tubular necrosis in a kidney transplant recipient," American Journal of Kidney Diseases, vol. 61, no. 5, pp. 782-785, 2013.

[39] H. R. Samimagham, S. Kheirkhah, A. Haghighi, and Z. Najmi, "Acute kidney injury in intensive care unit: incidence, risk factors and mortality rate," Saudi Journal of Kidney Diseases and Transplantation, vol. 22, no. 3, pp. 464-470, 2011.

[40] J. Y. Feng, K. T. Liu, E. Abraham et al., "Serum estradiol levels predict survival and acute kidney injury in patients with septic shock-a prospective study," PLoS One, vol. 9, no. 6, article e97967, 2014.

[41] N. Y. Kim, J. H. Hong, D. H. Koh, J. Lee, H. J. Nam, and S. Y. Kim, "Effect of diabetes mellitus on acute kidney injury after minimally invasive partial nephrectomy: a case-matched retrospective analysis," Journal of Clinical Medicine, vol. 8, no. 4, p. 468, 2019.

[42] J. Peng, X. Li, D. Zhang et al., "Hyperglycemia, p53, and mitochondrial pathway of apoptosis are involved in the susceptibility of diabetic models to ischemic acute kidney injury," Kidney International, vol. 87, no. 1, pp. 137-150, 2015.

[43] N. Abu-Saleh, D. Aronson, M. Khamaisi et al., "Increased intra-abdominal pressure induces acute kidney injury in an experimental model of congestive heart failure," Journal of Cardiac Failure, vol. 25, no. 6, pp. 468-478, 2019.

[44] J. Gameiro, M. Gonçalves, M. Pereira et al., "Obesity, acute kidney injury and mortality in patients with sepsis: a cohort analysis," Renal Failure, vol. 40, no. 1, pp. 120-126, 2018.

[45] J. Danziger, K. P. Chen, J. Lee et al., "Obesity, acute kidney injury, and mortality in critical illness," Critical Care Medicine, vol. 44, no. 2, pp. 328-334, 2016.

[46] J. P. van den Akker, M. Egal, and A. B. Groeneveld, "Invasive mechanical ventilation as a risk factor for acute kidney injury in the critically ill: a systematic review and meta-analysis," Critical Care, vol. 17, no. 3, p. R98, 2013.

[47] K. L. de Abreu, G. B. da Silva Junior, T. D. Muniz et al., "Acute kidney injury in critically ill patients with lung disease: kidneylung crosstalk," Rev Bras Ter Intensiva, vol. 25, no. 2, pp. 130136, 2013.
[48] B. Suberviola, E. Rodrigo, A. González-Castro, M. Serrano, M. Heras, and Á. Castellanos-Ortega, "Asociacion entre la exposicion previa a inhibidores de la enzima conversora de la angiotensina y los antagonistas de los receptores de la angiotensina en pacientes con fracaso renal agudo y shock septico," Medicina Intensiva, vol. 41, no. 1, pp. 21-27, 2017. 